$\mathrm{J}$

\title{
Atomic-Level Microstructure of Efficient Formamidinium-Based Perovskite Solar Cells Stabilized by 5 -Ammonium Valeric Acid lodide Revealed by Multinuclear and Two-Dimensional Solid-State NMR
}

Anwar Q. Alanazi, ${ }^{\dagger}$ Dominik J. Kubicki, ${ }^{\dagger},+, \bullet \odot$ Daniel Prochowicz, $^{\dagger, \S \odot ~ E s s a ~ A . ~ A l h a r b i, ~}{ }^{\dagger}$

Marine E. F. Bouduban, ${ }^{\perp}$ Farzaneh Jahanbakhshi, ${ }^{\nabla} \odot$ Marko Mladenović, $^{\nabla, \bigcirc}$ Jovana V. Milic, ${ }^{\dagger} \odot$

Fabrizio Giordano, ${ }^{\dagger}$ Dan Ren, ${ }^{\dagger} \odot$ Ahmed Y. Alyamani, Mohammad Hayal Alotaibi, ${ }^{\| \odot}$ Jacques-E. Moser, ${ }^{\perp} \odot$ Shaik M. Zakeeruddin, ${ }^{\dagger}$ Ursula Rothlisberger, ${ }^{\triangleright} \odot$

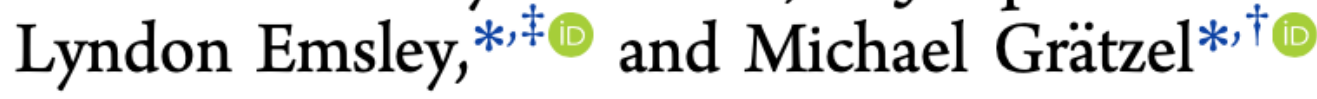

${ }^{\dagger}$ Laboratory of Photonics and Interfaces, Institute of Chemical Sciences and Engineering, School of Basic Sciences, Ecole Polytechnique Fédérale de Lausanne, CH-1015 Lausanne, Switzerland

${ }^{\ddagger}$ Laboratory of Magnetic Resonance, Institute of Chemical Sciences and Engineering, School of Basic Sciences, Ecole Polytechnique Fédérale de Lausanne, CH-1015 Lausanne, Switzerland

${ }^{\S}$ Institute of Physical Chemistry, Polish Academy of Sciences, Kasprzaka 44/52, 01-224 Warsaw, Poland

"National Center for Nanotechnology, King Abdulaziz City for Science and Technology, P.O. Box 6086, Riyadh 11442, Saudi Arabia ${ }^{\perp}$ Photochemical Dynamics Group, Institute of Chemical Sciences and Engineering, Centre for Ultrafast Science, École Polytechnique Fédérale de Lausanne CH-1015 Lausanne, Switzerland

\#Physics and Astronomy Department-Research Chair for Tribology, Surface and Interface Sciences, College of Science, and King Abdullah Institute for Nanotechnology-Aramco Laboratory for Applied Sensing Research, King Saud University, P.O. Box 2455, Riyadh 11451, Saudi Arabia

${ }^{\nabla}$ Laboratory of Computational Chemistry and Biochemistry (LCBC), École Polytechnique Fédérale de Lausanne (EPFL), CH-1015 Lausanne, Switzerland

OScientific Computing Laboratory, Center for the Study of Complex Systems, Institute of Physics Belgrade, University of Belgrade, Pregrevica 118, 11080 Belgrade, Serbia

S Supporting Information 